\title{
Especialización en Terapia Ocupacional: una oportunidad para el Perú
}

\author{
Specialization in Occupational Therapy: An opportunity for Peru \\ Carlos Manuel Escobar-Galindo ${ }^{1, a}$, Jesús Martin Trinidad-López ${ }^{1, a}$, Milagros de Jesús Céspedes-Chauca ${ }^{1, a}$, \\ Walter Miguel Reyes-Morales ${ }^{1, a}$, Edgar Cárdenas-Astete ${ }^{1, a}$
}

\section{RESUMEN}

Objetivo: Caracterizar la demanda actual de segunda especialización en Terapia Ocupacional en el Perú a través de las preferencias de los Terapeutas ocupacionales y las posibles áreas de interés. Material y métodos: El estudio fue descriptivo transversal. Se aplicó una encuesta virtual a los Terapeutas ocupacionales del Perú, respondiendo un total de 11 preguntas y completando un consentimiento informado. Estadísticos descriptivos y de asociación fueron utilizados. Resultados: Un total de 173 terapeutas ocupacionales devolvieron la encuesta completa de los cuales la gran mayoría fueron del género femenino (72,8\%). Así mismo, los participantes tenían más de 10 años de experiencia (47,9\%).Los Terapeutas ocupacionales prefirieron las especialidades de Pediatría (22,0\%) y Neurorrehabilitación (15,6\%). La especialidad de salud mental alcanzó el 13,9\%, seguido de Rehabilitación de Mano con 13,3\%, mientras que Educación el 8,7\%, siendo la de menor preferencia geriatría con el 3\%. Además, se encontró asociación entre el género y la elección de especialidades $(\mathrm{p}<0,05)$. Conclusiones: Las primeras especialidades de Terapia Ocupacional en ser creadas de acuerdo a las preferencias deberían ser Pediatría y Neurorrehabilitación. Las temáticas en cada una de las especialidades merecen un análisis especifico. Si bien hay preferencias entre los profesionales de Terapia ocupacional es necesario desarrollar estrategias claras que permitan la creación de especialidades basadas en las necesidades del país y que faciliten la inserción de los terapeutas ocupacionales a los diversos equipos, pero no solo de salud si no también a diferentes ámbitos de actuación comunitario y social

PALABRAS CLAVE: Terapia Ocupacional; especialización; Pediatría; neurorrehabilitación.

\section{SUMMARY}

Objective: To characterize the current preference of second specialization in Occupational Therapy and the possible areas of interest according to the reality of Peru. Material and Methods: The study was descriptive, cross-sectional. A virtual survey was applied to occupational therapists in Perú. A total of 11 questions were responded and signed an informed consent. Descriptive and association statistics were used. Results: A total of 173 occupational therapists returned the survey of which the great majority were female $(72.8 \%)$. Likewise, the participants had more than 10 years of experience (47.9\%), Occupational therapists preferred the specialities of Paediatrics (22.0\%) and Neurorehabilitation (15.6\%). Mental health speciality reached 13.9\%, followed by Hand Rehabilitation with $13.3 \%$, while Education $8.7 \%$, being geriatrics the least preferred with $3 \%$. Furthermore, an association was found between gender and specialities $(\mathrm{p}<0.05)$. Conclusions: According to preferences of occupational therapists first specialties to be created according should be Paediatrics and Neurorehabilitation. The topics in each of the specialties deserve specific analysis. There are specialties that are not registered in Peruvian legislation, but they are of high priority for

\footnotetext{
1 Escuela de Tecnología Médica, Facultad de Medicina, Universidad Peruana Cayetano Heredia. Lima, Perú

a Terapeuta Ocupacional .
} 
the country, such as Occupational Therapy in community and related to prevention and social areas. However, all specialties must cover not only biomedical approach but also preventive and social aspects.

KEY WORDS: Occupational Therapy, specialization, Paediatrics, neurorehabilitation.

\section{INTRODUCCIÓN}

La Terapia Ocupacional en Latinoamérica ha ido evolucionando favorablemente. El incremento de la esperanza de vida (1), el aumento de la población con discapacidad $(2,3)$ y con problemas de salud mental (4), entre otros incrementan la necesidad de profesionales como los Terapeutas Ocupacionales para promover el bienestar de la población a través de un óptimo desempeño ocupacional. En Perú la Terapia Ocupacional es una carrera profesional que lleva aproximadamente 50 años de fundada siendo la Universidad Nacional Mayor de San Marcos (UNMSM) pionera en la formación de terapeuta ocupacionales. Luego, a partir del año 1998 la Universidad Peruana Cayetano Heredia (UPCH) se sumó a la formación de profesionales en Terapia Ocupacional. Sin embargo, la cantidad de escuelas formadoras es aún muy limitada para cubrir las necesidades a nivel nacional de profesionales en Terapia Ocupacional. Los profesionales egresados llevan el título profesional de Tecnólogo Médico en el área o la especialidad de Terapia Ocupacional, denominación utilizada para referirse a los profesionales de Terapia Ocupacional en el Perú debido a que forma parte del colegio profesional de Tecnólogos Médicos del Perú (CTMP) (5). Si bien la carrera de Terapia Ocupacional tiene una identidad propia reconocida a nivel internacional, en el Perú está incluida desde sus orígenes dentro del área de la Tecnología Médica siendo requisito legal para ejercerla aunque su campo profesional va más allá del ámbito biomédico o de la salud $(6,7)$. De acuerdo al último censo de profesionales publicada por el CTMP (8), los terapeutas ocupacionales representan el $2 \%$ de la población de Tecnólogos Médicos en el Perú, constituyendo la más baja proporción con respecto a las demás especialidades. Además, el censo señala que la mayor cantidad son del género femenino $(57.78 \%)$, ubicándose gran parte de la población entre los 30 y 50 años (66\%). Esta evolución de la carrera a lo largo de los años ha llevado a que muchos de los profesionales en Terapia Ocupacional desarrollen competencias específicas en diversos aspectos de su quehacer profesional. Actualmente los profesionales han estado capacitándose a nivel de maestría y doctorado en diversas áreas de acción. Cerca del 92\% de terapeutas ocupacionales encuestados han llevado una maestría y un $8 \%$ doctorado sin titularse lo que refleja la necesidad de capacitación de los profesionales para desempeñarse en sus diversas áreas, sin embargo estos programas de posgrado no son directamente relacionados en Terapia Ocupacional. Muchas de las competencias logradas a nivel de posgrado han servido como insumo base para fortalecer sus respectivas áreas de desempeño desarrollando notablemente los campos de trabajo de la Terapia Ocupacional. Sin embargo, estas competencias son reconocidas principalmente a nivel académico y formativo docente, pero no en sus respectivos lugares de trabajo, por tanto, la denominación de especialista en un área determinada se vuelve imperativo como mecanismo legal para el reconocimiento y fortalecimiento de una competencia profesional de especialista.

La especialización es un proceso de la educación que tiene como objetivo ampliar los conocimientos y las concepciones del área, la preparación de profesionales para intervenir y transformar la práctica. El especialista se dedica a una rama de su profesión de acuerdo con su capacidad o interés (9). La evolución histórica de la Terapia Ocupacional y los cambios sociodemográficos de la población han demostrado la necesidad de estructurar el conocimiento para lograr la eficacia de las intervenciones, es por eso que Moruno formula un esquema que enfatiza un modelo de práctica y abordaje donde se establecen puentes entre lo teórico y práctico para establecer técnicas, modalidades, medios, métodos y procedimientos de evaluación y tratamiento que se utilizarán en encuadres concretos (10). En ese contexto la creación de especialidades en Terapia Ocupacional aporta significativamente sobre la evolución filosófica y científica de encuadrar un modelo teórico para lograr la eficiencia de los resultados (10).

Como antecedente, la Terapia Ocupacional cuenta con una oferta de posgrado en países de Latino América como Brasil, Chile, Colombia y México; en donde las especialidades de mayor coincidencia fueron neonatal y pediátrica, clínica, salud mental y cuidados paliativos (11). En el Perú, el CTMP por medio de la resolución 0206-2016 reconoció diez posibles especialidades en Terapia Ocupacional bajo diferentes denominaciones indicando que el tiempo mínimo de 
formación es de un año y un máximo de dos, previo reconocimiento de la Superintendencia Nacional de Educación Superior (SUNEDU) (12). De acuerdo a la actual Ley Universitaria, las especialidades en el Perú deben tener una duración mínima de un año y medio cumpliendo un creditaje de al menos 40 créditos académicos y deben ser impartidas por docentes con grado de maestría registrada (13). A pesar de existir mecanismos legales claros que aseguran la posibilidad de desarrollar programas formativos de especialidad, en la actualidad ninguna de las universidades formadoras en Terapia Ocupacional en el Perú tiene oferta educativa a nivel de posgrado. Es evidente ante la actual coyuntura la necesidad de crear estos programas formativos , por lo que es necesario atender las opiniones de los terapeutas ocupacionales peruanos acerca de las preferencias sobre los programas de segunda especialidad teniendo como base la resolución del CTMP sobre el registro de segundas especialidades , así como también proyectar futuras especializaciones necesarias para el desarrollo de la carrera y garantizar un óptimo bienestar de la población a través de una adecuada participación ocupacional.

Por tanto, el presente trabajo tuvo como objetivo caracterizar la demanda actual de una segunda especialidad en Terapia Ocupacional en el Perú a través de las preferencias de los terapeutas ocupacionales y las posibles áreas de interés necesarias para crear programas de especialización requeridas para el país.

\section{MATERIAL Y MÉTODOS}

El estudio fue descriptivo de corte transversal basado en el análisis de la población de terapeutas ocupacionales en el Perú.

Para definir la muestra, se tomó en cuenta una proyección de un universo aproximado de 308 terapeutas ocupacionales habilitados en el Perú basado en la información disponible del CTMP (8). Considerando este población se realizó un cálculo muestral por proporciones para población finita, calculando con un nivel de confianza del $95 \%$, y una proporción esperada del 50\%, un mínimo de 171 personas.

Para realizar el estudio se diseñó una encuesta virtual cuyas preguntas fueron definidas de acuerdo a los objetivos trazados en el estudio. Las respuestas por ningún motivo requirieron de algún tipo de información de carácter confidencial o privado. El número total de preguntas fue de 11 de las cuales 3 fueron preguntas abiertas y el resto cerradas. Se redujeron de 13 a 11 preguntas siguiendo las recomendaciones de otros estudios para reducir la probabilidad del bajo ratio de respuestas en encuestas virtuales (14). El tiempo promedio para completar la encuesta fue de aproximadamente cinco minutos medidos en un estudio piloto previo de cinco participantes. Con respecto a las preferencias de especialidad se toma en consideración la resolución $\mathrm{N}^{\circ}$ 0206-CTMP-CN/2016 del CTMP acerca de las segundas especialidades registradas (12), además de la actual oferta de especialidades en el mercado internacional. Sin embargo, se deja una pregunta abierta con la finalidad de que los participantes puedan establecer posibles áreas de especialidad no mencionadas.

La encuesta fue anónima de tal forma que se mantuvieron el respeto de la autonomía de los informantes, además los participantes tuvieron toda la libertad de poder retirarse del estudio o dejar inconclusa la encuesta si así lo estimaban conveniente. La encuesta fue aplicada de manera online a través del servidor Bristol Online Survey (BOS). El servidor generó un link que fue compartido en un grupo de trabajo virtual constituido por más de 220 Terapeutas ocupacionales del Perú. La encuesta se pudo completar por medio del uso de celulares o computadoras con conexión a internet lo cual facilitó su aplicación. El periodo de aplicación de la encuesta fue durante el mes de enero del 2020. Solo se consideraron en el conteo final las encuestas que fueron completadas en su totalidad.

Los datos finales de la encuesta fueron exportadas a una hoja de cálculo de Microsoft Excel 2019 ${ }^{\mathrm{TM}}$ en donde la información fue codificada y organizada para luego llevarlo al programa estadístico SPSS v.24 (IBM). Se realizó un análisis descriptivo de los datos mostrando las variables cualitativas en tablas de frecuencia y proporciones mientras que las variables numéricas fueron ordenadas en promedios y desviación estándar. Para establecer asociaciones entre especialización y variables sociodemográficas se utilizó el chi cuadrado de independencia. Se estableció como valor de significancia el 5\% $(\mathrm{p}<0.05)$

Para procesar lo datos de las preguntas abiertas, los autores del presente artículo que son terapeutas ocupacionales con amplia experiencia, organizaron la información y agruparon las respuestas en temas específicos que puedan abarcar la temática explicada de tal forma que se represente mediante un gráfico resumen con los temas específicos. 
Tabla 1. Datos sociodemográficos de los participantes

\begin{tabular}{lcc}
\hline \multicolumn{1}{l}{ Sexo } & $\mathbf{n}$ & $\mathbf{\%}$ \\
\hline Masculino & 47 & 27,2 \\
Femenino & 126 & 72,8 \\
Edad & & \\
$<30$ años & 56 & 32,4 \\
30 a 50 años & 111 & 64,2 \\
$>50$ años & 6 & 3,5 \\
Tiempo de egresado & & \\
$<1$ año & 7 & 4,0 \\
1 a 5 años & 48 & 27,7 \\
5 a 10 años & 32 & 18,5 \\
$>10$ años & 86 & 49,7 \\
\hline
\end{tabular}

Tabla 2. Distribución de la muestra de acuerdo a la formación y grado académico

\begin{tabular}{lcc}
\hline & $\mathbf{n}$ & $\mathbf{\%}$ \\
\hline Universidad de procedencia & & \\
$\begin{array}{l}\text { Universidad Nacional Mayor de } \\
\text { San Marcos }\end{array}$ & 157 & 90,8 \\
$\begin{array}{l}\text { Universidad Peruana Cayetano } \\
\text { Heredia }\end{array}$ & 16 & 9,2 \\
Grado académico & & \\
Bachiller & 39 & 22,5 \\
Licenciado & 117 & 67,6 \\
Magister & 13 & 7,5 \\
Doctor & 0 & 0,0 \\
\hline
\end{tabular}

\section{RESULTADOS}

En total se recolectaron 177 encuestas de las cuales 173 fueron consideradas como válidas puesto que completaron el cuestionario en su totalidad. La edad promedio de los participantes fue de 35 años $(7,8 \%)$ siendo el grupo de 30 a 50 años el más numeroso $(64,2 \%)$. La mayor cantidad de participantes fueron del género femenino $(72,8 \%)$, y egresados con más de 10 años $(49,7 \%)$ (tabla 1$)$.

Los profesionales principalmente provienen de la UNMSM $(90,8 \%)$ y en menor porcentaje de la UPCH $(9,2 \%)$. El $67.6 \%$ tienen el título de licenciado y grado académico de bachiller, mientras que el $22.5 \%$ solo cuenta con el grado académico de bachiller. Apenas un $7,5 \%$ de los participantes tiene el grado académico de magíster y ninguno de doctor (tabla 2).

Con respecto al centro de labores de los participantes, el 38\% labora en un consultorio privado, el $23 \%$ en hospitales y centros de salud del Ministerio de Salud (MINSA), el 8\% en hospitales del seguro social del Perú (ESSALUD) ,8\% en el sector Educación (colegios regulares y especiales), el 7\% en centros de salud de las Fuerzas Armadas ,7\% Instituciones Privadas , 5\% en clínicas, 3\% en instituciones del Programa Integral Nacional para el Bienestar Familiar (INABIF) y apenas $1 \%$ en Programas de Atención Temprana nacionales (PRITE) (gráfico 1).

La especialidad de Terapia Ocupacional en Pediatría tuvo mayor porcentaje alcanzando casi un

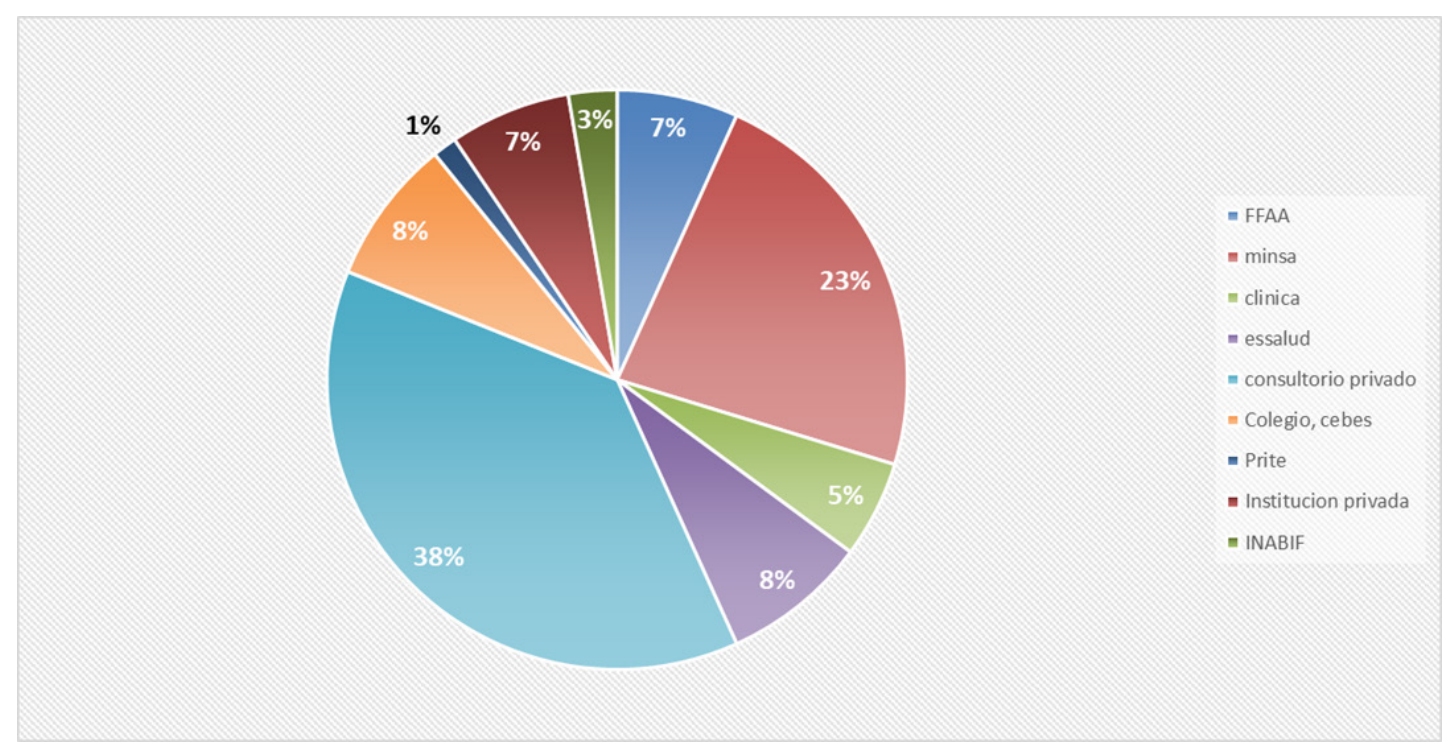

Grafico 1. Distribución de la muestra en función al centro de labores. 
cuarto del total de las preferencias $(22,0 \%)$, seguido de Terapia Ocupacional en Neurorrehabilitación $(15,6 \%)$. La especialidad de Terapia Ocupacional en Salud Mental y Rehabilitación de Manos y Miembro superior alcanzaron el 13,9\%, mientras que Terapia Ocupacional en Educación el 8,7\%. Los resultados en las demás especialidades fueron menores al $5 \%$ (figura 1). Si en caso se fusionaran las especialidades

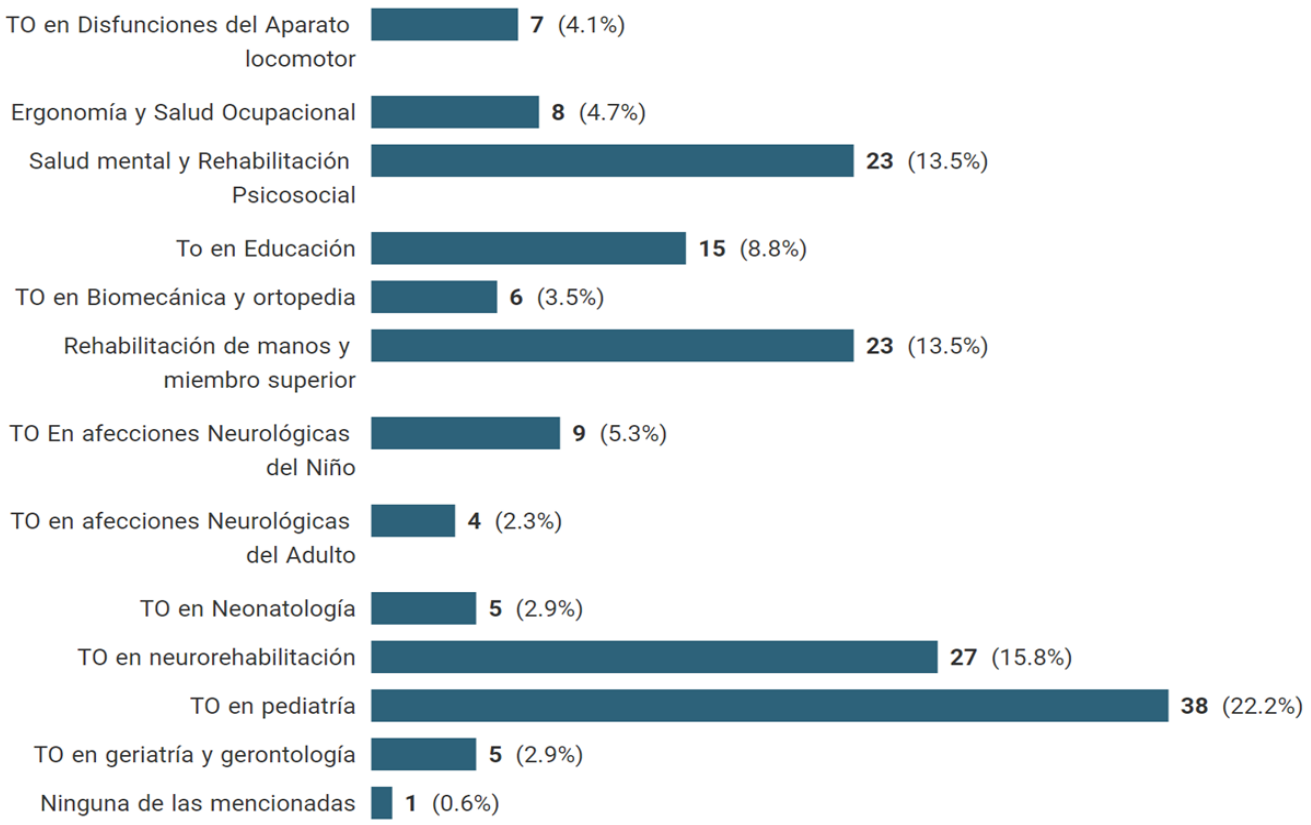

Gráfico 2. Distribución de las preferencias de segunda especialidad entre Terapeutas Ocupacionales

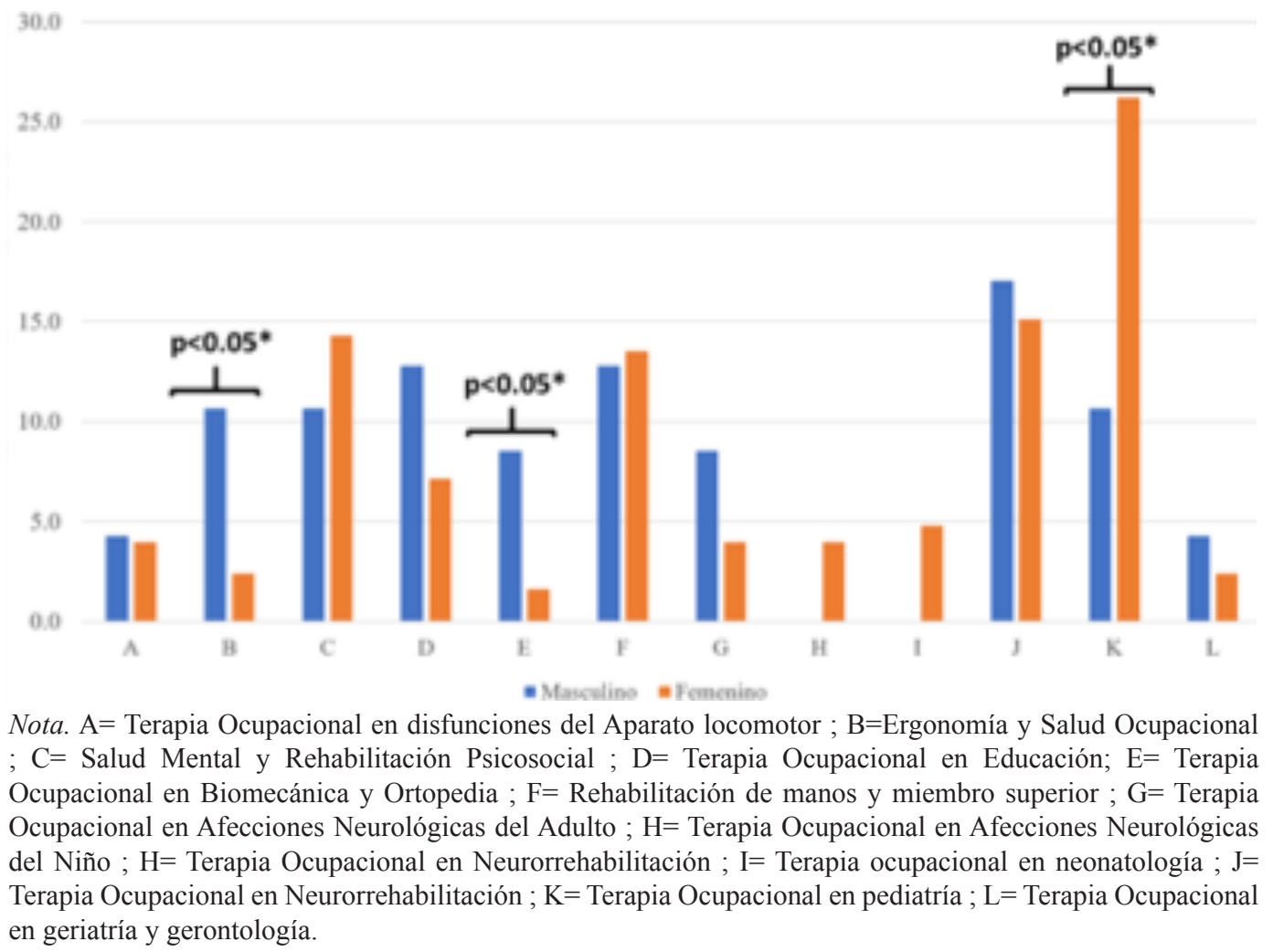

Grafico 3. Asociación de especialidades con sexo de los participantes. 
relacionadas a niños y adultos, la especialidad de Terapia Ocupacional en Pediatría y Terapia Ocupacional en Neurorrehabilitación alcanzarían $30 \%$ y $23 \%$ de las preferencias respectivamente. En caso se fusionaron las especialidades relacionados a disfunciones físicas de adultos y biomecánica, la especialidad de rehabilitación de mano alcanzaría el $21 \%$ de las preferencias (Gráfico 2).

La especialidad de Terapia Ocupacional en Pediatría está altamente asociada al género femenino $(\mathrm{p}<0.05)$ mientras que las especialidades de Ergonomía y Salud ocupacional y Biomecánica y Ortopedia están asociadas al género masculino $(\mathrm{p}<0,05)$. Las demás especialidades no tienen asociación al género. Además, tampoco se observa asociación con la universidad de procedencia, grado académico, ni con el tiempo de egreso (gráfico 3).

Entras las diversas especialidades sugeridas por los participantes, se evidenció repetición en varias de ellas, concluyendo finalmente en nueve especialidades que no fueron mencionadas en el estudio. Entre las principales especialidades se encuentra, Terapia Ocupacional en Oncología. , Terapia Ocupacional en la Comunidad, Terapia Ocupacional en Educación Especial, Terapia Ocupacional en Integración Sensorial entre otros (gráfico 4).

\section{DISCUSIÓN}

La encuesta refleja lo que es evidente en la práctica mundial, el alto porcentaje de mujeres en la práctica profesional de la Terapia Ocupacional la cual "tradicionalmente estuvo dominado por mujeres"(15) with strategies proposed to recruit more men.Purpose. This article explores whether this is a legitimate equity concern.Key Issues.Most employment is gender segregated; when gender balances change, the field either re-genders feminine or creates gendersegregated internal divisions. Men avoid feminized jobs because they pay less and hold less social status. They are a ?step down? for men. In such jobs, men are disproportionately pushed into management positions, with better pay, more prestige, and less hands-on care. Equity issues concern structural barriers to success in particular employment fields. Though they may feel discomfort in a feminized field, men do not face structural barriers in occupational therapy. Implications.Broader challenges to traditional gender norms are needed, but there is no evidence that gender parity is an equity concern or that recruitment targeting masculinity would make a difference.","containertitle":"Canadian Journal of Occupational Therapy".

Sin embargo, es notorio el incremento en el número de varones en la profesión (8). Este aumento de varones

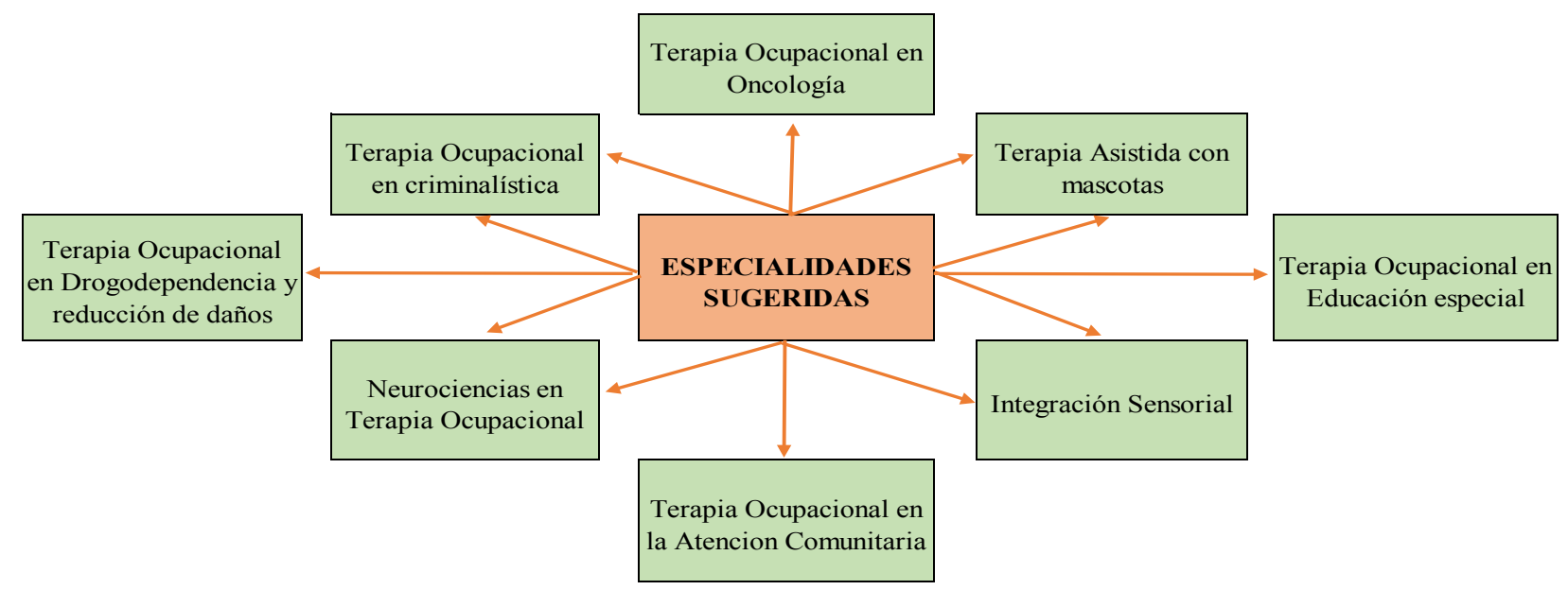

Gráfico 4. Especialidades sugeridas no mencionadas por los Terapeutas Ocupacionales 
en cierto modo tiene influencia en las posibles áreas de especialización que son preferentes entre los varones más que en las mujeres. Por ejemplo, la encuesta señala que los varones tienen mayor preferencia por la especialidad de Ergonomía y Salud Ocupacional y Biomecánica y ortopedia, probablemente debido a que requieren de mayor esfuerzo físico o cuyo campo de acción está más ligado al trabajo y al diseño. Con respecto al grado académico se observa que solo el $13 \%$ tiene maestría con grado académico registrado y ninguno con doctorado lo que en cierta manera contradice lo reflejado en el censo del CTMP (8), por lo que la información debe ser considerada con suma cautela.

Las preferencias sobre la especialidad entre los terapeutas ocupacionales están principalmente en el campo de la Pediatría y de la Neurorrehabilitación sin desmerecer el porcentaje de preferencia de las otras especialidades. Con respecto a las preferencias por género las mujeres tienen una alta afinidad por la especialidad de pediatría, probablemente debido a la alta demanda de profesionales en esta área y el reducido número de terapeutas disponibles.

El periodo de la infancia es una etapa de desarrollo muy crítico desde el aspecto físico, psíquico y social debido a que en esta etapa el ser humano madura neurológicamente . Los padres prestan mucha atención e invierten en la formación general de sus niños especialmente en lo relacionado al desarrollo psicomotriz y sensorial por lo que la demanda de trabajar en esta especialidad es mayor. La expansión del trabajo con niños está siendo aprovechada por un buen número de profesionales de la Terapia Ocupacional que justamente ven en la necesidad del mercado una gran oportunidad para empoderarse dentro de las organizaciones tanto en el ámbito de la salud como en el de la educación. Esta coyuntura está favoreciendo el desarrollo e interés en el área el cual se ve reflejada en la encuesta. Sin embargo, la temática relacionada a pediatría se expande aún más que el trabajo con niños con alguna enfermedad o discapacidad puesto que en este espacio se tendría que diferenciar el área de Terapia Ocupacional en Educación regular y/o especial, así como el periodo de la adolescencia. A pesar de la alta preferencia y gran demanda, la actual legislación no contempla la especialización de Pediatría como parte de las especializaciones en Terapia Ocupacional, si no lo define como un grupo de especialización que incluye neonatología, afecciones neurológicas en niños y educación (12).
Los desórdenes neurológicos constituyen una de las principales causas de mortalidad en el mundo llegando hasta un $16.8 \%$ de las muertes en países de Latinoamérica, y aquellas personas que sobreviven deben vivir permanente con una secuela neurológica que afecta su calidad de vida. Se estima que las tasa de secuelas por problemas neurológicos serán de aproximadamente 14\% al 2030 (3). Estas cifras alertan sobre la necesidad de actuar sobre los usuarios que tienen discapacidad por secuela neurológica y que por tanto requieren de una intervención especializada para lograr su reinserción social y laboral. En la actualidad los terapeutas ocupacionales se deben capacitar en el extranjero tomando cursos de especialización individuales sin certificación universitaria, para acreditar sus competencias en este campo. Sin embargo, no existen oportunidades donde se puedan adquirir habilidades y competencias necesarias para fortalecer esta especialización. La creación de una especialización en Neurorrehabilitación abrirá oportunidades necesarias para crear estrategias especificas en pacientes con desórdenes neurológicos de todas las edades enfocados a maximizar su independencia y optimizar su desempeño en la vida diaria.

Por su parte la salud mental está adquiriendo relevancia en la salud pública por la gran demanda de soluciones a los problemas sociales relacionados a la violencia, ansiedad y depresión en los diferentes niveles socioeconómicos del país (4) . En respuesta a esta necesidad, el Estado Peruano recientemente ha promulgado el Reglamento de la Ley de Salud Mental N 30947 (17), el cual tiene un alcance de aplicación en los ámbitos preventivo, promocional, curativo, rehabilitador y de reinserción social. Esto nos muestra un interés político sobre la necesidad de empoderar a los profesionales encargados de la salud mental dentro de los niveles del Sistema de Salud para trabajar sobre estas necesidades. Como antecedente histórico la terapia Ocupacional nació desde el trabajo de salud mental a inicios del siglo 20 promoviendo la desinstitucionalización de individuos con problemas mentales, desde ahí es que ahora se fomenta la práctica de la Terapia Ocupacional a las comunidades (18). Diversos estudios son referenciados demostrando la evidente eficacia de la Terapia Ocupacional en los problemas de salud mental siendo parte indispensable del equipo multidisciplinario de rehabilitación psicosocial y en la comunidad (19).

Terapia ocupacional en geriatría es la especialidad que obtuvo menor preferencia por parte de los 
terapeutas ocupacionales en Perú, evidenciando ello una incongruencia entre la inclinación de los profesionales y la necesidad de la sociedad. Esta afirmación se basa en dos condiciones, la primera relacionada al proceso de transición demográfica y el incremento en la esperanza de vida generando que en un periodo corto de tiempo la población de este grupo etario será la predominante (1); la segunda referida a la cantidad reducida de establecimientos de salud públicos y privados para el abordaje de los adultos mayores; esta segunda condición es la que hace que la oferta de trabajo sea reducida generando que el terapeuta elija otra especialidad en la que cuente con mejores opciones laborales.

La evolución natural de las nuevas demandas sociales y políticas no puede divorciarse de la necesidad de que los colegios profesionales realicen actualizaciones permanentes en la formulación de sus especialidades luego de evaluaciones periódicas de un equipo técnico por cada especialidad. De acuerdo a la actual legislación (12), se describen especialidades que no necesariamente se alinean a las nuevas necesidades y demandas sociales que el país requiere. Lo cierto es que las bases de la Terapia Ocupacional tienen una connotación más amplia que va más allá de los aspectos biomédicos abarcando no solo actuaciones en el ámbito hospitalario o asistencial si no también a nivel de educativo, laboral y social comunitario $(6,7))$. Prueba de ello son las diferentes recomendaciones que los profesionales realizan para crear especialidades relacionados a aspectos relacionados a la coyuntura actual tales como: criminalística, drogodependencia, educación especial, comunidad entre otros. Por lo que se hace necesario reestructurar la propuesta basado en un análisis prospectivo en el ámbito de la salud pública, educación, sociolaboral y desarrollo comunitario.

\section{CONCLUSIONES}

La especialización de Terapia ocupacional en Pediatría y en Neurorrehabilitación fueron las que alcanzaron los mayores porcentajes de preferencia, por lo que puede ser tomado como punto inicial para el desarrollo de especializaciones. Sin embargo, si bien hay preferencias entre los profesionales de Terapia ocupacional lo cierto es que es necesario desarrollar estrategias claras que permitan la creación de especialidades basadas en las necesidades del país y que faciliten la inserción del Terapeuta Ocupacional a los diversos equipos, pero no solo de salud sino también a diferentes ámbitos de actuación tales como, Comunidad, laboral, criminalística entre otros. Los Terapeutas ocupacionales han expresado claramente su opinión con respecto a las posibilidades de especialización y han confirmado la tendencia evolutiva de la profesión. Basado en los resultados se deben introducir en todas las especialidades el carácter preventivo y promocional de la carrera para optimizar el desempeño ocupacional saludable que permita a los individuos gozar de una participación ocupacional digna y con justicia social.

\section{Correspondencia:}

Manuel Escobar

Correo electrónico: manoergo@gmail.com

\section{REFERENCIAS BIBLIOGRAFICAS}

1. Instituto Nacional de Estadística e Informática. Esperanza de vida de población peruana aumentó en 15 años en las últimas cuatro décadas . Lima: Instituto Nacional de Estadística e Informática; 2015. Disponible en: http://m.inei.gob.pe/media/ MenuRecursivo/noticias/nota_de_prensa_n188 2015inei_2.pdf

2. Instituto Nacional de Estadística e Informática. Encuesta Nacional Especializada sobre Discapacidad 2012. Lima: Instituto Nacional de Estadística e Informática; 2014 p. 588. Disponible en: https:// www.inei.gob.pe/media/MenuRecursivo/ publicaciones_digitales/Est/Lib1171/ENEDIS\%20 2012\%20-\%20COMPLETO.pdf

3. World Health Organization. Neurological Disorders: Public Heatlh challenges. Ginebra: World Health Organization; 2006.

4. Rondón MB. Salud mental: un problema de salud pública en el Perú. Revista Peruana de Medicina Experimental y Salud Publica. 2006; 23:237-8.

5. Congreso de la República del Perú. Ley Profesional del Tecnólogo Médico. $\mathrm{N}^{\circ}$ 28456. Lima: Diario Oficial El Peruano; 20 de diciembre del 2004. Disponible en: http://files.servir.gob.pe/WWW/files/ normas\%20legales/Ley\%2028456.pdf

6. Guajardo A, Maria S. Critical Reflections on human rights: Contributions from the Latin American Occupational Therapy. World Federation of Occupational Therapists Bulletin. 2015;71(2):73-80. Doi: https://doi.org/10.179/1447382815Z.000000000 23

7. Pan L. Políticas de ensino superior, graduação em terapia ocupacional e o ensino de terapia ocupacional social no Brasil. Tesis de Grado. Belo Horizonte, Brasil: Universidade Federal de Minas Gerais; 2014. Disponible en: https://repositorio.ufscar.br/handle/ ufscar/6889

8. Colegio Tecnólogo Medico del Perú. Primer Censo Nacional de Tecnólogos Médicos del Perú. Lima: 
Colegio Tecnólogo Médico del Perú; 2015.

9. Paula M, Santos L. O significado de ser especialista para o enfermeiro estomaterapeuta. Revista LatinoAmericana de Enfermagem. 2003;11:474-82.

10. Moruno P, Talavera M. Estructurar el conocimiento clave en la práctica de la terapia ocupacional. TOG (A Coruña). 2009;6(9):0-0. Disponible en: http:// www.revistatog.com/num9/pdfs/modelo.pdf

11. Gómez S, Imperatore E. Desarrollo de la Terapia Ocupacional en Latinoamérica. Revista Chilena de Terapia Ocupacional. 2010; 10:123-135. DOI: 10.5354/0719-5346.2010.10566

12. Colegio Tecnólogo Medico del Perú. Registro Nacional de Especialidad Profesional en Tecnología Médica. Lima: Colegio Tecnologo Medico del Perú; 2016.

13. Congreso de la República del Perú. . Ley universitaria del Perú. 30220. Lima: Ministerio de Educacion; 2014. Disponible en: http://www.minedu.gob.pe/ reforma-universitaria/pdf/ley_universitaria.pdf
14. Park A, Lee G, Seagull FJ, Meenaghan N, Dexter D. Patients benefit while surgeons suffer: an impending epidemic. Journal of the American College of Surgeons. 2010; 210(3):306-13.

15. Beagan BL, Fredericks E. What about the men? Gender parity in occupational therapy: Qu'en est-il des hommes? La parité hommes-femmes en ergothérapie. Can J Occup Ther. 2018;85(2):137-45.

16. Rosselli M. Maduración cerebral y desarrollo cognoscitivo. Revista Latinoamericana de Ciencias Sociales, Niñez y Juventud. 2003;1(1):125-44.

17. Ministerio de Salud. Reglamento de Ley de Salud mental No 30947. Lima: Ministerio de Salud; 2020.

18. Precin P. A Review of: "Occupational Therapy in Mental Health: Considerations for Advanced Practice, edited by Marian Kavanaugh Scheinholtz". Occupational Therapy in Mental Health. 2011;27(2):191-3.

19. Brown C. Occupational therapy practice guidelines for adults with serious mental illness. Bethesda, $\mathrm{Md}$ : American Occupational Therapy Association; 2012. 